\title{
Antibiotic use in children hospitalised with pneumonia in Central Vietnam
}

\author{
Phuong TK Nguyen, ${ }^{1,2}$ Hoang T Tran, ${ }^{3}$ Dominic A Fitzgerald, ${ }^{2,4}$ Steve M Graham, ${ }^{5,6}$ \\ Ben J Marais ${ }^{2}$
}

\begin{abstract}
- Additional material is
published online only. To view please visit the journal online (http://dx.doi.org/10.1136/ archdischild-2019-317733).
\end{abstract}

${ }^{1}$ Department of Respirology, Da Nang Hospital for Women and Children, Da Nang, Vietnam ${ }^{2}$ Discipline of Paediatrics and Adolescent Medicine, The University of Sydney, Sydney, New South Wales, Australia ${ }^{3}$ Department of Neonatology, Da Nang Hospital for Women and Children, Da Nang, Vietnam ${ }^{4}$ Respiratory Medicine, Children's Hospital at

Westmead, Sydney, New South Wales, Australia

${ }^{5}$ Department of Paediatrics, Centre for International Child Health, University of Melbourne, Melbourne, Victoria, Australia ${ }^{6}$ Murdoch Children's Research Institute, Royal Children's Hospital, Melbourne, Australia, Melbourne, Victoria, Australia

Correspondence to Dr Phuong TK Nguyen, Respirology, Da Nang hospital for Women and Children, Da Nang 55000, Viet Nam; thng5150@uni.sydney.edu.au

Received 13 June 2019 Revised 11 December 2019 Accepted 22 January 2020 Published Online First 20 February 2020

Check for updates

(C) Author(s) (or their employer(s)) 2020. No commercial re-use. See rights and permissions. Published by BMJ.

To cite: Nguyen PTK, Tran HT, Fitzgerald DA, et al.

Arch Dis Child

2020;105:713-719.

\section{ABSTRACT}

Background and objectives Excessive use of antibiotics has been noted in children with respiratory tract infections in Vietnam, but antibiotic use in hospitalised children is poorly documented. Antibiotic use and direct healthcare costs in children hospitalised with pneumonia in central Vietnam were assessed.

Methods A prospective descriptive study of children under 5 years old admitted with a primary admission diagnosis of 'pneumonia' to the Da Nang Hospital for Women and Children over 1 year.

Results Of 2911 children hospitalised with pneumonia, 2735 (94.0\%) were classified as 'non-severe' pneumonia by the admitting physician. In total, 2853 (98.0\%) children received antibiotics. Intravenous antibiotics were given to $336(12.3 \%)$ children with 'non-severe' and 157/176 (89.2\%) children with 'severe' pneumonia; those with 'non-severe' pneumonia accounted for $68.2 \%$ (336/493) of intravenous antibiotics given. Only 19.3\% (95/493) of children on intravenous antibiotics were stepped down to an oral antibiotic. Cefuroxime was the preferred oral agent, and ceftriaxone was the preferred injectable agent. Hospital admission for oral antibiotics in 'non-severe' pneumonia was a major cost driver, with an average direct cost of US\$78.9 per patient, accounting for $54.0 \%$ of the total hospitalisation cost in the study cohort. In addition, 336 (12.3\%) children with non-severe pneumonia received intravenous antibiotics without indication, accounting for a further $23.2 \%$ of hospitalisation costs.

Conclusion Limiting unnecessary hospitalisation and considering early intravenous to oral step down antibiotic will reduce direct health system costs and morbidity in children with respiratory tract infections in Vietnam.

\section{INTRODUCTION}

Pneumonia is the most important cause of underfive morbidity and mortality outside the neonatal period. ${ }^{1}$ Despite advances in diagnostic tests, improved immunisation coverage and standardised case management approaches, pneumonia was responsible for more than 600000 deaths in children under-five in 2016, mostly in developing countries. ${ }^{2}$ Appropriate antibiotic treatment is a key weapon to reduce deaths in children with bacterial pneumonia. ${ }^{3}$ However, the inappropriate use of antibiotics contributes to antimicrobial resistance and is associated with multiple adverse effects, including negative impacts on the microbiome and increased healthcare costs. ${ }^{4}$ Encouraging rational antibiotic prescription is a global priority, particularly in Asia, where childhood pneumonia is a major

\section{What is already known on this topic?}

- Childhood pneumonia is a major contributor to antibiotic use and hospital admissions in Vietnam and other East Asian countries.

- Inappropriate use of antibiotics contributes to antimicrobial resistance and is associated with multiple adverse effects.

- Encouraging more rational antibiotic use is a global priority, particularly in Asia, where access to antibiotics is largely unrestricted.

\section{What this study adds?}

- Unnecessary hospitalisation for childhood pneumonia is a major healthcare cost driver in Vietnam, likely also in other East Asian countries, but this is often sustained by systemic healthcare factors beyond physicians' control.

- Early step down from intravenous to oral antibiotics is rarely practised and could further reduce healthcare cost and other adverse effects associated with unnecessary hospital stay.

- Routine use of broad-spectrum antibiotics is preferred to oral amoxicillin but is generally not associated with improved outcome.

driver of antibiotic use and where access to antibiotics is largely unrestricted. ${ }^{45}$

Compliance with national and international pneumonia guidelines can be improved with better provider training, diagnostic capacity and awareness of local antibiotic drug susceptibility patterns. $^{67}$ Guidelines typically recommend oral amoxicillin as first-line treatment for non-severe pneumonia and benzylpenicillin for hospitalised children with uncomplicated severe pneumonia. ${ }^{8-11}$ The use of broad-spectrum antibiotics, mostly later generation cephalosporins and beta-lactam/ beta-lactamase combinations, is generally reserved for initial empiric therapy in critically ill patients or when the presence of penicillin-resistant pathogens is suspected or preferably confirmed. ${ }^{811}$ Nonsevere pneumonia can be managed as an outpatient with a 5-day course of oral antibiotics. ${ }^{1012}$ Despite international recommendations and published evidence to support this approach, broad-spectrum antibiotics continue to be overprescribed, ${ }^{413}$ while unnecessary hospital admission and extended 
treatment durations impose a huge financial burden on families and healthcare systems. ${ }^{14-16}$

In Vietnam, pneumonia accounts for nearly a third of all paediatric admissions to hospital. ${ }^{15} 1718$ Indications are that many of these 'pneumonia' admissions do not meet WHO criteria for pneumonia and that even less meet strict criteria for severe pneumonia, ${ }^{15}{ }^{16}$ which generally guides the need for hospital admission and the use of intravenous antibiotics. Childhood pneumonia is a major contributor to antibiotic use and hospital admissions in other East Asian countries as well. ${ }^{17-19}$ Since there are limited data on in-hospital antibiotic use, we aimed to describe the profile of children hospitalised with pneumonia, focusing on place of admission, type/route and duration of antibiotic administration, with calculation of the associated direct healthcare cost.

\section{MATERIAL AND METHODS}

We conducted a prospective descriptive study of all children hospitalised with 'pneumonia', as diagnosed by the admitting physician, over a 1-year study period (1 July 2017 to 30 June 2018)

\section{Study setting}

The Da Nang Hospital for Women and Children is a referral provincial hospital in central Vietnam with 570 paediatric beds (for children $<15$ years old). Children with pneumonia are admitted to either the respiratory ward (140 beds) or the intensive care unit (ICU; 30 beds) depending on the severity of disease, as assessed by the admitting physician. There is limited microbiological support to guide antibiotic treatment, and blood culture is not routinely done. Initial treatment may be adjusted by senior clinicians if they believe the treatment is inadequate (usually within $<3$ days) or if treatment response is suboptimal (usually after $\geq 3$ days).

In 2015, the Vietnam Ministry of Health released a childhood pneumonia guideline ${ }^{11}$ in which amoxicillin was listed as the first-line antibiotic of choice, with amoxicillin/clavulanic acid, cefuroxime, cefaclor, erythromycin and azithromycin listed as second-line alternatives. Antibiotics were recommended for a minimum period of 5 days, with intravenous antibiotics reserved for children who experience a pneumonia-related complication (eg, empyema) or poor response to oral antibiotics. Intravenous to oral step down antibiotic was advised following adequate clinical recovery and once oral antibiotics were tolerated. Children under 6 years old in Vietnam receive free healthcare insurance, which covers hospitalisation cost but pay a maximum of US $\$ 10$ for outpatient services. Additional expenses for consultation, blood tests, chest radiograph and drugs are directly payable by the caregivers if the child is not admitted to hospital. The minimum service fee for an outpatient clinic visit and receiving 5 days of a first-line oral antibiotic is US\$3.1.

\section{Study population and classification}

We recruited children aged 2-59 months with a primary admission diagnosis of 'pneumonia'. We excluded children admitted to a private (fee-paying) ward, referred from other hospitals or admitted with a secondary diagnosis of pneumonia. Independent researchers performed daily rounds to recruit new patients. Recorded information included: antibiotic use before admission (type, duration and antibiotic provider), clinical signs/symptoms, medical history, social risk factors and antibiotics given at presentation. Data related to the treatment course (type and duration of antibiotics given), pneumonia outcome and hospitalisation cost were finalised on discharge.

'Non-severe' and 'severe' pneumonia were classified by the admitting clinician who did not necessarily follow the revised WHO classification. In Vietnam, any child with respiratory symptoms and fever or audible crackles is usually diagnosed with 'pneumonia', with a subjective severity assessment based on tachypnoea, chest indrawing, nasal flaring, grunting, cyanosis or lung infiltrates on the chest radiograph. A previous analysis of the study cohort demonstrated that a minority of admissions met WHO criteria for 'severe pneumonia'. ${ }^{16}$ In this analysis, we focus on in-hospital antibiotic use and direct hospital cost, which were not previously assessed.

Antibiotic treatment was classified as 'single oral' if a child received only one oral antibiotic until discharge; 'addition of second oral' if an additional oral antibiotic was added during ward review by a senior clinician; 'oral IV switch' if intravenous antibiotics (for at least 2 days) were initiated after admission with oral antibiotics; 'IV only' if the child received intravenous antibiotics for the full period of hospitalisation; 'IV oral step down' if an oral antibiotic was given (for at least 1 day) before hospital discharge in a child who previously received intravenous antibiotics.

Direct hospital cost included all medical costs associated with the hospital admission, including initial examination fee, bed occupancy and daily review (ICU bed costs three times more than a ward bed), investigations and procedures, drugs used in hospital (patients pay separately for prescriptions at discharge), oxygen supply, physical therapy and medical devices (needles, nebuliser, mechanical ventilation and so on).

\section{Statistical analysis}

Data were entered into an Epi Data (V.4.4.3.1) database. Initial data were entered within a day of admission with final data entry and checking completed on hospital discharge. Data were carefully checked for inconsistencies and corrections made by referring to the original patient. Data analysis was carried out using SPSS (V.24.0). We performed simple descriptive analyses to provide an overview of the cohort. Continuous data were summarised as mean (SD) or median (IQR). For comparative analyses, we considered unrecorded data as missing values and did not use any imputation. Categorical data were compared using $\chi^{2}$ or Fisher's exact tests, as appropriate. A p value $\leq 0.05$ was considered statistically significant.

\section{RESULTS}

Of the 2911 children admitted with pneumonia, 2778 (95.4\%) were admitted to the respiratory ward, $87(3.0 \%)$ to the ICU and $46(1.6 \%)$ were first admitted to the respiratory ward and later transferred to the ICU (online supplementary figure S1). Figure 1 reflects the duration of antibiotic use relative to the route of administration and disease severity (as assessed by the admitting clinician). Nearly all $(2853 ; 98.0 \%)$ children received antibiotics; $2360(81.1 \%)$ received oral antibiotics only and 493 $(17.0 \%)$ received intravenous antibiotics. This included most children with 'severe' pneumonia (157/176; 89.2\%), although the majority of intravenous antibiotic recipients (336/493; $68.2 \%$ ) had 'non-severe' pneumonia. Few children received 'IV to oral step down' and usually only after completion of 7 days of intravenous antibiotics. Children with 'non-severe' pneumonia received a median of 6 days of antibiotics and those with 'severe' disease a median of 7 days. The longest median hospital stay (12 days) occurred in children with oral to intravenous switch after 
Recommendation by national guideline ${ }^{\#}$

Duration of in-hospital antibiotic use $(2853,98 \%)$
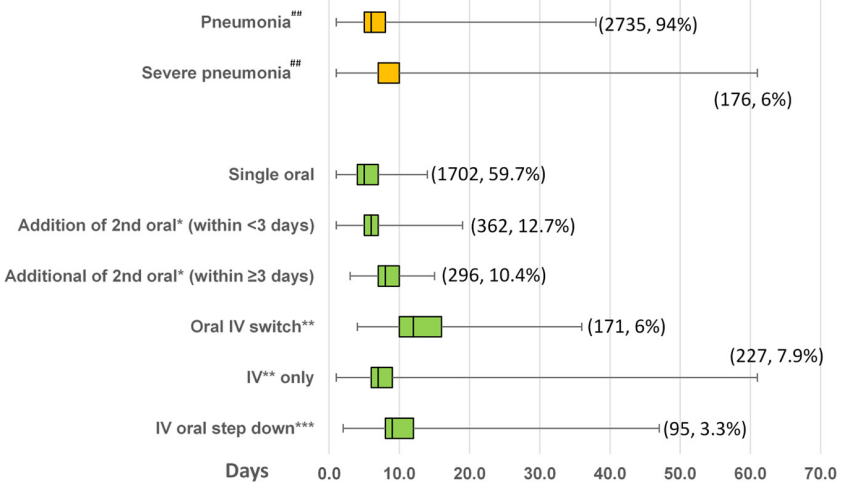

Figure 1 Duration of in-hospital antibiotic use according to pneumonia severity ${ }^{\# \#}$ and route of administration (relative number; \%); whisker plot presents values from left-hand side to right-hand side in order: min, 25\% IQR, median, 75\% IQR, max.

IV - intravenous; IQR - interquartile range

${ }^{a}$ Most children completed their full course of antibiotics in-hospital; antibiotics given in hospital are generally free of charge for all children under 5 years of age, which is not the case for home prescriptions; "referring to the Vietnamese guideline for the management of community-acquired pneumonia in children; antibiotics are generally recommended for 5 days, ${ }^{11 ~ \# \#}$ as classified by the admitting clinician; median of severe pneumonia was 7 days (25\% IQR 7-10 days); ${ }^{*}$ a second oral antibiotic (usually a macrolide) added after initial consultant review (if $<3$ days and) or following no perceived treatment response (if $\geq 3$ days); **including ampicillin ( \pm sulbactam), cefotaxime, ceftriaxone, ceftazidime, cefoperazone ( \pm sulbactam), vancomycin, imipenem/ cilastatin, ticarcillin ( \pm clavulanic acid), linezolid, levofloxacin, colistin, meropenem, metronidazole and piperacillin ( \pm tazobactam); ***at least 1 day of oral antibiotic before hospital discharge.

hospital admission. These children experienced either prolonged wheeze, typical of viral bronchiolitis or worsening symptoms due to a potential secondary infection while in hospital.

Table 1 reflects differences in demographic and clinical characteristics between children admitted to the respiratory ward and the ICU. Children admitted to the ICU were younger with an over-representation of preterm and low birthweight babies. ICU admissions presented more commonly with chest in-drawing or 'danger signs', while children admitted to the respiratory ward had a higher frequency of 'runny nose' and audible wheeze. Of 87 children admitted to the ICU, 46 (52.9\%) remained in hospital for more than 10 days, compared with 10.5\% (291/2778) admitted to the respiratory ward. Online supplementary table S1 shows that most children $(1462,50.2 \%)$ received antibiotics before hospital admission.

Table 2 compares the type of antibiotic used and the total duration of treatment in children admitted to either the respiratory ward or the ICU. Among 83 children with wheeze and no fever at presentation, $81(97.6 \%)$ received an antibiotic. Clinicians generally preferred oral cefuroxime or intravenous cefotaxime as first-line treatment. Table 3 summarises the treatment changes observed with the use of different first-line oral antibiotics given in hospital. Around 10\% (139/1436, 9.7\%) of children treated with cefuroxime were switched to an intravenous antibiotic during hospitalisation, which was higher than that observed with amoxicillin and amoxicillin/clavulanic acid
(7.3\% and 5.0\%, p<0.001). Children started on cefuroxime had similar pneumonia severity classification and risk of being admitted to ICU as those given amoxicillin or amoxicillin/ clavulanic acid. Macrolide use was associated with the most frequent use of an 'add-on' oral $(641 / 871,73.6 \%)$ or intravenous $(116 / 871,13.3 \%)$ antibiotic.

Table 4 summarises the total direct hospitalisation cost and cost per hospitalised day in children admitted with pneumonia, according to disease severity and antibiotic treatment received. The admission of children with 'non-severe' pneumonia for oral antibiotic delivery costs US\$12.3 per day (average of US\$78.9 per patient), accounting for $54.0 \%$ of the total hospitalisation cost for the study cohort. The cost for 5 days of oral antibiotics given as an outpatient would have been US\$3.1. In addition, 336 $(12.3 \%)$ children with non-severe pneumonia received intravenous antibiotics without clinical indication, accounting for a further $23.2 \%$ of the total hospitalisation cost. Most children completed a 'full course' of intravenous antibiotics, with only $19.3 \%$ (95/493) stepping down to oral antibiotics in hospital. The highest cost among children not initially admitted to the ICU was documented in the group of children who experienced a switch from oral to intravenous administration after hospital admission: US\$245.8 for 'non-severe' pneumonia and US\$1194.6 for 'severe' pneumonia.

\section{DISCUSSION}

We found high numbers of children admitted to hospital with 'non-severe' pneumonia, especially when more objective WHO classification criteria were used. ${ }^{16}$ Hospital admission for the provision of oral antibiotics, which could have been provided on an outpatient basis, was a major contributor to healthcare cost in the study cohort. However, for children less than 6 years of age, this cost is not borne by families but by the national health insurance scheme. In fact, although outpatient care is cheaper and safer than hospital admission for children with non-severe pneumonia, the direct cost borne by families for outpatient care is higher. This is a major incentive for unnecessary hospitalisation, and families are often unaware of the risks associated with hospital admission or the cost to the healthcare system. In addition, many children with 'non-severe' pneumonia received intravenous antibiotics, further increasing healthcare cost and adverse events associated with intraenous-line management. Among those started on intravenous antibiotics, step-down to oral antibiotics was uncommon, which is inconsistent with international practice and WHO guidelines.

Systemic incentives to hospitalise children with non-severe conditions is a major challenge throughout Southeast Asia, ${ }^{4} 20$ with children in China often receiving routine intravenous antibiotics when hospitalised with community-acquired pneumonia. ${ }^{19}$ This is different to findings from sub-Saharan Africa where most children hospitalised with pneumonia have severe disease. ${ }^{22} 23$ Apart from increased cost, hospitalisation also increases the risk of hospital-acquired infection without outcome benefit in children with non-severe pneumonia. ${ }^{12}$ Revised WHO pneumonia case definitions and management guidelines greatly reduced the number of children requiring hospital admission, ${ }^{24}$ but these guidelines have not been widely adopted in Vietnam. Our findings suggest that there is huge potential to reduce healthcare cost and patient risk by improving pneumonia management practices. ${ }^{16}$ The money saved from limiting unnecessary hospitalisation could be used to increase pneumonia prevention through pneumococcal vaccination or other enhanced services if supported by the funding model. 
Table 1 Demographic and clinical characteristics of children admitted to the respiratory ward and intensive care unit (ICU) with a primary diagnosis of pneumonia*

\begin{tabular}{|c|c|c|c|c|c|}
\hline \multirow[b]{2}{*}{ Demographics and clinical characteristics } & \multicolumn{2}{|c|}{ Respiratory wardt } & \multicolumn{2}{|l|}{ ICU } & \multirow[b]{2}{*}{$P$ value } \\
\hline & $\begin{array}{l}\text { Pneumonia } \\
(n=2705)\end{array}$ & $\begin{array}{l}\text { Severe pneumonia* } \\
(n=119)\end{array}$ & $\begin{array}{l}\text { Pneumonia } \\
(\mathrm{n}=30)\end{array}$ & $\begin{array}{l}\text { Severe pneumonia* } \\
(n=57)\end{array}$ & \\
\hline Age group (months) & & & & & $<0.001$ \\
\hline $2-11$ & $763(28.2)$ & $78(65.5)$ & $17(56.7)$ & $38(66.7)$ & \\
\hline $12-23$ & $1076(39.8)$ & $28(23.5)$ & $5(16.7)$ & $15(26.3)$ & \\
\hline $24-59$ & $866(32.8)$ & $13(10.9)$ & $8(26.7)$ & $4(7.0)$ & \\
\hline Male & $1553(57.4)$ & $63(52.9)$ & $19(63.3)$ & $36(63.2)$ & 0.3 \\
\hline Cough & $2541(93.9)$ & $106(89.1)$ & $23(76.7)$ & $49(86.0)$ & $<0.001$ \\
\hline Runny nose & $670(24.8)$ & $21(17.6)$ & $4(13.3)$ & $5(8.8)$ & 0.001 \\
\hline Any fever & $2205(81.5)$ & $76(63.9)$ & $20(66.7)$ & $40(70.2)$ & 0.009 \\
\hline Chest indrawing & $203(7.5)$ & $69(58.0)$ & $15(50.0)$ & $44(77.2)$ & $<0.001$ \\
\hline Grunting & $18(0.7)$ & $14(11.8)$ & $6(20.0)$ & $7(12.3)$ & $<0.001$ \\
\hline Nasal flaring & $10(0.4)$ & $9(7.6)$ & $6(20.2)$ & $8(14.0)$ & $<0.001$ \\
\hline Audible wheeze & $198(7.3)$ & $16(13.4)$ & 0 & $3(5.3)$ & $0.3 \S$ \\
\hline Wheeze on auscultation & $195(7.2)$ & $12(10.1)$ & $5(16.7)$ & $7(12.3)$ & 0.02 \\
\hline Crackles & $2094(77.4)$ & $111(93.3)$ & $24(80.0)$ & $48(84.2)$ & 0.5 \\
\hline Tachypnoea & $1311(48.5)$ & $97(81.5)$ & $19(63.3)$ & $43(75.4)$ & $<0.001$ \\
\hline SpO2 $<90 \%$ & $10 / 154(6.5)$ & $24 / 63(38.1)$ & $8 / 15(53.3)$ & $21 / 46(47.5)$ & $<0.001$ \\
\hline \multicolumn{6}{|l|}{ Medical history and social risk factors } \\
\hline ARI admission last 12 months & $1053(39.0)$ & $35(29.4)$ & $14(46.7)$ & $25(43.9)$ & 0.2 \\
\hline ARI readmission 2 weeks & $277(30.2)$ & $15(12.6)$ & $5(16.7)$ & $12(21.1)$ & 0.02 \\
\hline Tuberculosis contact & $56(2.1)$ & $6(5.0)$ & 0 & $2(3.5)$ & $1.0 \S$ \\
\hline Any breast feeding & $2508(92.9)$ & $106(89.1)$ & $27(90.0)$ & $48(84.2)$ & 0.08 \\
\hline Pneumococcal vaccineף & $113(4.2)$ & $4(3.9)$ & $1(3.3)$ & $3(5.3)$ & $0.8 \S$ \\
\hline Daycare attendance & $1517(56.5)$ & $30(25.2)$ & $5(16.7)$ & $8(14.0)$ & $<0.001$ \\
\hline Sibling ill & $510(18.7)$ & $34(28.6)$ & $5(16.7)$ & $15(26.3)$ & 0.4 \\
\hline Cigarette smoke exposure & $1407(52.1)$ & $62(52.1)$ & $19(63.3)$ & $33(57.9)$ & 0.2 \\
\hline Preterm ( $<37$ weeks) & $247(9.0)$ & $20(16.8)$ & $3(10.0)$ & $12(21.1)$ & $<0.001$ \\
\hline Low birth weight $(<2500 \mathrm{~g})$ & $188(6.8)$ & $20(16.8)$ & $3(10.0)$ & $10(17.5)$ & 0.01 \\
\hline \multicolumn{6}{|l|}{ Outcome } \\
\hline Oxygen supplementation & $74(2.0)$ & $50(42.0)$ & $27(90.0)$ & $51(91.1)$ & $<0.001$ \\
\hline Hospital stay $>10$ days & $288(10.4)$ & $20(16.8)$ & $17(56.7)$ & $29(50.9)$ & $<0.001$ \\
\hline Hospital cost** (USD) & 89.0 & 129.8 & 490.4 & 532.8 & $<0.001$ \\
\hline
\end{tabular}

*Pneumonia severity assessment, as recorded by the admitting physician.

†Patients admitted to the respiratory ward and then transferred to the ICU were included among 'respiratory ward' patients in the comparative analysis.

fUsing the $\chi^{2}$ test to compare all patients (pneumonia and severe pneumonia combined) admitted to the respiratory department versus those admitted to the ICU.

§Using the Fisher's exact test.

IPneumococcal conjugate vaccine.

**Including examination fee on admission, bed occupancy, investigations and procedures, drugs, oxygen supply, physical therapy and medical devices.

ARI, acute respiratory infection; SpO2, peripheral oxygen saturation.

A previous detailed assessment of this cohort found that a large proportion of patients did not fulfil WHO pneumonia criteria, which was supported by chest radiograph and peripheral blood test analysis that was essentially normal. ${ }^{25}$ Given limited experience with WHO pneumonia management guidelines and low pneumococcal vaccination rates in Vietnam, many physicians are uncomfortable to rule out bacterial pneumonia, although respiratory viruses are the most commonly isolated respiratory pathogens. ${ }^{26-28}$ In this cohort, children with wheeze and no fever at presentation, who do not require antibiotics according to revised WHO recommendations, ${ }^{29}$ still received antibiotics. Most guidelines advise the use of amoxicillin as first-line antibiotic in nonsevere pneumonia. ${ }^{910}$ However, cefuroxime, a second generation cephalosporin, was often prescribed as standard practice without any disease severity or microbiological consideration. Given the assumption that most children would already have received antibiotics by the time they present to hospital, most doctors prefer using broad-spectrum antibiotics, although no benefit has been demonstrated in adults or children with community-acquired 'pneumonia'. ${ }^{30}$ Luckily, the use of third generation oral cephalosporins was limited, unlike other Asian settings where its use is common. 3233

A recent survey in Vietnam confirmed excellent penicillin susceptibility in Streptococcus pneumoniae isolated from respiratory specimens, while cephalosporin and macrolide susceptibility was poor. ${ }^{33}$ This suggests that a high dose of amoxicillin remains an excellent antibiotic choice in paediatric pneumonia cases. Oral antibiotics are frequently used in combination in Asian countries, with almost a third of hospitalised children receiving macrolides and cephalosporins. ${ }^{13} 19$ This is often given to cover for atypical organisms such as Mycoplasma pneumoniae, but high rates of macrolide resistance recorded in multiple settings ${ }^{4}$ suggests futility and unnecessary cost. Our data showed that macrolide use was associated with the highest rate of oral to 
Table 2 Comparison of antibiotic use in children admitted to either the respiratory ward or the ICU with pneumonia

\begin{tabular}{|c|c|c|c|c|c|}
\hline \multirow[b]{2}{*}{ In-hospital antibiotic use } & \multicolumn{2}{|c|}{ Respiratory ward* $(n=2824)$} & \multicolumn{2}{|c|}{ ICU $(n=87)$} & \multirow[b]{2}{*}{$P$ valuet } \\
\hline & $\mathrm{N}(\%)$ & Days (median, IQR) & $\mathrm{N}(\%)$ & Days (median, IQR) & \\
\hline Antibiotic used & $2767(98.0)$ & $6.0(5.0-8.0)$ & $86(98.9)$ & $8.0(7.0-14.0)$ & $>0.05$ \\
\hline \multicolumn{6}{|l|}{ Oral antibiotic only $^{d}$} \\
\hline Total & $\mathrm{n}=2354$ & - & $n=6$ & - & \\
\hline Cefuroxime $\ddagger$ & $1297(55.1)$ & $6.0(4.0-7.0)$ & $3(50.0)$ & $3.0(2.0-4.0)$ & $>0.05 \S$ \\
\hline Amoxicillin & $39(1.7)$ & $5.0(4.0-7.0)$ & 0 & - & $>0.05 \S$ \\
\hline Amoxi/clav & $973(41.3)$ & $5.0(4.0-7.0)$ & $1(16.7)$ & 5.0 & $>0.05 \S$ \\
\hline Macrolide & $752(32.0)$ & - & $3(50.0)$ & - & \\
\hline Erythromycin & $39(1.7)$ & $6.0(4.0-8.0)$ & $2(33.3)$ & 6.0 & $<0.05 \S$ \\
\hline Azi/clarithromycin & $713(30.3)$ & $5.0(4.0-5.0)$ & $1(16.7)$ & 5.0 & $>0.05 \S$ \\
\hline \multicolumn{6}{|c|}{ Intravenous antibiotic only" } \\
\hline Total & $n=180$ & - & $\mathrm{n}=47$ & - & \\
\hline Ampicillin & $65(36.1)$ & $7.0(6.0-7.0)$ & $10(21.3)$ & $7.0(4.8-8.0)$ & $>0.05$ \\
\hline $\begin{array}{l}\text { Cephalosporin third } \\
\text { generation }\end{array}$ & $110(61.1)$ & - & $36(76.6)$ & - & \\
\hline Cefotaxime & $80(44.4)$ & $7.0(6.0-8.0)$ & $21(44.7)$ & $7.0(6.5-9.5)$ & $>0.05$ \\
\hline Ceftriaxone & $28(15.6)$ & $8.0(7.0-10.0)$ & $6(12.8)$ & $6.0(4.5-10.0)$ & $>0.05$ \\
\hline Ceftazidime & $2(1.1)$ & $8.5(8.0-9.0)$ & $9(19.1)$ & $8.0(7.0-12.0)$ & $<0.001$ \\
\hline Vancomycin & $8(4.4)$ & $10.0(7.3-16.0)$ & $8(17.0)$ & $14.0(9.3-19.8)$ & $<0.01$ \\
\hline Clindamycin & 0 & - & $2(4.3)$ & $15.0(10.0-20.0)$ & $<0.05 \S$ \\
\hline Imipenem/cilastatin & $3(1.7)$ & $10.0(8.0-12.0)$ & $6(12.8)$ & $15.5(14.0-21.0)$ & $<0.01$ \\
\hline Ticarcillin & $4(2.2)$ & $9.5(7.5-13.8)$ & 0 & - & $>0.05 \S$ \\
\hline Otherq & $5(2.8)$ & $6.0(4.0-8.8)$ & $3(6.5)$ & $9.0(8.0-13.5)$ & $>0.05 \S$ \\
\hline \multicolumn{6}{|c|}{ Intravenous and then oral antibiotic } \\
\hline Total & $\mathrm{n}=71$ & $9.0(8.0-12.0)$ & $n=24$ & $11.0(8.0-18.0)$ & - \\
\hline \multicolumn{6}{|c|}{ Oral and then intravenous antibiotic } \\
\hline Total & $n=162$ & $12.0(10.0-15.0)$ & $n=9$ & $23.0(13.0-29.5)$ & - \\
\hline
\end{tabular}

*Patients admitted to the respiratory ward and then transferred to the ICU were included among 'respiratory ward' patients in the comparative analysis.

tUsing $\chi^{2}$ test to compare proportions between departments.

¥Mostly cefuroxime (second-generation cephalosporin), with only limited third-generation (cefixime) use.

§Using Fisher's extract test; the total add to more than $\mathrm{N}(>100 \%)$, since some children received more than one antibiotic at the same time.

IIncluding ampicillin+sulbactam, cefoperazone, ticarcillin+clavulanic acid, ciprofloxacin, levofloxacin, linezolid, colistin, meropenem, metronidazole, piperacillin \pm tazobactam; antivirals and antifungals were not considered.

Amoxi/clav, amoxicillin with clavulanic acid; azi, azithromycin; ICU, intensive care unit.

intravenous switch or using an 'add-on' oral antibiotic. Unnecessary hospitalisation and antibiotic use has also been documented in China, ${ }^{13}$ even after confirmation of a likely viral infection, ${ }^{19}$ with intravenous cephalosporin use in $>90 \%$ of cases. ${ }^{13}$

Most children received a longer antibiotic course than recommended by national and international guidelines. This has also been observed in multiple retrospective studies in Vietnam ${ }^{15} 21$ and China. ${ }^{13}$ In the study setting, children generally completed a full course of antibiotics (either oral or intravenous) before hospital discharge, since this is provided free of charge to all children $<6$ years of age. Few children were discharged on oral antibiotics, which partly reflect standard practice and parent expectation. However, there is little regard for the risks and costs (direct and indirect) associated with unnecessary hospital stay and strong evidence that oral amoxicillin given at home provides adequate treatment for non-severe pneumonia. ${ }^{34}$ We calculated the direct healthcare cost according to the actual treatment received, irrespective of its appropriateness, which reflects the

Table 3 Treatment changes according to first-line oral antibiotic given for childhood pneumonia at hospital admission

\begin{tabular}{|c|c|c|c|c|}
\hline \multirow[b]{2}{*}{ Treatment changes (outcome) } & \multicolumn{4}{|c|}{ First-line oral antibiotic given in hospital } \\
\hline & Amoxicillin & Amoxi/clav & Cefuroxime* & Macrolide \\
\hline No add-on antibiotic given & $26(63.4)$ & $744(72.7)$ & $859(59.8)$ & $114(13.1)$ \\
\hline 'Add-on' oral antibiotic within $<3$ daysł & $4(9.8)$ & $118(11.5)$ & $242(16.9)$ & $354(40.6)$ \\
\hline 'Add-on' oral antibiotic after $\geq 3$ days $\ddagger$ & $8(19.5)$ & $111(10.8)$ & $196(13.6)$ & $287(33.0)$ \\
\hline Switch to intravenous antibiotics & $3(7.3)$ & $51(5.0)$ & $139(9.7)$ & $116(13.3)$ \\
\hline Total & 41 & 1024 & 1436 & 871 \\
\hline
\end{tabular}

*The decision to give cefuroxime instead of amoxicillin is usually not based on disease severity, but we were unable to rule out potential unmeasured bias in the patient cohorts. tIncluding erythromycin, azithromycin and clarithromycin.

¥Addition of either a macrolide (if not on a macrolide) or a beta-lactam antibiotic (if on a macrolide) post admission during ward review by a senior clinician (rarely based on microbiology results).

Amoxi/clav, amoxicillin with clavulanic acid. 
Table 4 Direct hospital cost ${ }^{\#}$ calculated by antibiotic treatment used during hospitalisation for children with a primary diagnosis of pneumonia or severe pneumonia

\begin{tabular}{lcccc}
\hline & & \multicolumn{2}{l}{ Direct hospital cost (US\$)* } & \\
\cline { 2 - 5 } Antibiotic treatment received & Average cost/episode & Average cost/day & Total cost for the study cohort (\%) \\
\hline 'Non-severe' pneumonia & $2735(94.0)$ & 96.7 & 12.9 & $266475(78.0)$ \\
\hline Oralt antibiotic only & $2341(85.6)$ & 78.9 & 12.3 & $184705(54.0)$ \\
\hline Intravenous antibiotic only & $114(4.2)$ & 237.3 & 18.3 & $27052(7.9)$ \\
\hline Oral intravenous switch & $166(6.1)$ & 245.8 & 16.8 & $40803(11.9)$ \\
\hline Intravenous oral step down & $56(2.0)$ & 205.1 & 17.9 & $11486(3.4)$ \\
\hline No antibiotic received & $58(2.1)$ & 49.7 & 11.6 & $2883(0.8)$ \\
'Severe' pneumonia & $176(6.0)$ & 429.6 & 20.3 & $75610(22.0)$ \\
\hline Oralt antibiotic only & $19(10.8)$ & 115.8 & 15.3 & $2200(0.6)$ \\
\hline Intravenous antibiotic only & $113(64.2)$ & 493.4 & 20.6 & $55754(16.3)$ \\
\hline Oral intravenous switch & $5(2.8)$ & 1194.6 & 37.7 & $5973(1.7)$ \\
\hline Intravenous oral step down $\neq$ & $39(22.2)$ & 299.8 & 19.8 & $11692(3.4)$ \\
\hline
\end{tabular}

*Including examination fee on admission, bed occupancy, investigations and procedures, drugs, oxygen supply, physical therapy and medical devices.

tSingle or combination of oral antibiotics.

₹At least 1 day of oral antibiotic before hospital discharge.

cost of real-life practice. Providing 7 days of intravenous treatment essentially reflects established practice, ${ }^{10}$ although an early intravenous to oral switch policy reduces hospital stay, secondary complications and overall healthcare costs. ${ }^{3637}$ Prolonged administration of intravenous antibiotics remains common practice in many Asian countries. ${ }^{13} 19$ An educational programme in Indonesia reduced unnecessary intravenous antibiotic use, ${ }^{38}$ but it required ongoing interaction with clinicians to sustain its impact.

In conclusion, this prospective study presents important 'reallife' data that provide enhanced insight into the antibiotic treatment of child pneumonia cases in Vietnam. Clinicians generally preferred broad-spectrum cephalosporins and frequently added a macrolide as a second antibiotic, despite the fact that few children had signs of severe disease. In addition, most children who received intravenous antibiotics were not considered to have severe pneumonia. We identified unnecessary hospitalisation and reluctance to consider intravenous to oral step down with home discharge on oral antibiotics, as major drivers of healthcare cost and nosocomial disease transmission risk.

Acknowledgements We would like to thank all patients and caregivers for their participation in the study. We are especially grateful to three respiratory ward nurses (Oanh TK Nguyen, Nhung TM Le and Ut T Doan) who helped with data collection on admission. We acknowledge support from the Da Nang government who provided a PhD scholarship to the first author (PTKN). This manuscript is in partial fulfilment of a PhD thesis.

Contributors PTKN, SMG and BJM conceptualised the study and designed the protocol. PTKN collected the data and drafted the manuscript. PTKN and BJM analysed the data. All authors reviewed and approved the final manuscript.

Funding PTKN holds a Da Nang government PhD scholarship and work at the Da Nang Hospital for Women and Children.

Competing interests None declared.

Patient consent for publication Not required.

Provenance and peer review Not commissioned; internally peer reviewed.

Data availability statement Data are available on reasonable request. The first author (PTKN) are holding original data. Please email her at thng5150@uni.sydney. edu.au for data sharing request.

\section{REFERENCES}

1 Walker CLF, Rudan I, Liu L, et al. Global burden of childhood pneumonia and diarrhoea. Lancet 2013;381:1405-16.

2 Troeger C, Blacker B, Khalil IA, et al. Estimates of the global, regional, and national morbidity, mortality, and aetiologies of lower respiratory infections in 195 countries,
1990-2016: a systematic analysis for the Global Burden of Disease Study 2016. Lancet Infect Dis 2018:18:1191-210.

3 Shann F. Etiology of severe pneumonia in children in developing countries. Pediatr Infect Dis J 1986:5:247-52.

4 Phuong NTK, Hoang TT, Van PH, Nguyen TKP, Tran TH, Pham HV, et al. Encouraging rational antibiotic use in childhood pneumonia: a focus on Vietnam and the Western Pacific region. Pneumonia 2017:9:7

5 WHO. Antimicrobial resistance: global report on surveillance, 2014. Available: http:// wwwwhoint/drugresistance/documents/surveillancereport/en/ [Accessed 5 Dec 2019]

6 Izadnegahdar R, Cohen AL, Klugman KP, et al. Childhood pneumonia in developing countries. Lancet Respir Med 2013;1:574-84.

7 Graham Set al. Challenges to improving case management of childhood pneumonia at health facilities in resource-limited settings. Bull World Health Organ 2008;86:349-55.

8 WHO. Pocket book of hospital care for children: guidelines for the management of common childhood illnesses. World Health Organization, 2013.

9 WHO. Revised WHO classification and treatment of childhood pneumonia at health facilities. Geneva: World Health Organization, 2014

10 Nascimento-Carvalho AC, Nascimento-Carvalho CM. Clinical management of community-acquired pneumonia in young children. Expert Opin Pharmacother 2019:20:435-42.

11 Viet Nam Ministry of Health. Guideline for antibiotic using (in Vietnamese). Ha Noi: Medical Journal, 2015

12 Addo-Yobo E, Chisaka N, Hassan M, et al. Oral amoxicillin versus injectable penicillin for severe pneumonia in children aged 3 to 59 months: a randomised multicentre equivalency study. Lancet 2004;364:1141-8.

$13 \mathrm{Mi}$, Li W, Zhang L, et al. The drug use to treat community-acquired pneumonia in children: a cross-sectional study in China. Medicine 2018;97:e13224.

14 Zhang S, Sammon PM, King I, et al. Cost of management of severe pneumonia in young children: systematic analysis. J Glob Health 2016;6:010408.

15 Nguyen TKP, Nguyen DV, Truong TNH, et al. Disease spectrum and management of children admitted with acute respiratory infection in Viet Nam. Trop Med Int Health 2017;22:688-95.

16 Nguyen PTK, Tran HT, Fitzgerald DA, et al. Characterisation of children hospitalised with pneumonia in central Vietnam: a prospective study. Eur Respir J 2019;54:1802256.

17 Nelson EAS, Tam JS, Yu LM, et al. Assessing disease burden of respiratory disorders in Hong Kong children with hospital discharge data and linked laboratory data. Hong Kong Med J 2007;13:114.

18 Zhang $X$, Zhang J, Cao A. [Epidemiological investigation on respiratory diseases in 1300 children, in Jinan, Shandong]. Zhonghua Liu Xing Bing Xue Za Zhi 2014;35:1275-7.

19 Wei W, Wang XF, Liu JP, et al. Status of antibiotic use in hospitalized children with community-acquired pneumonia in multiple regions of China. Zhonguo Dang Dai Er Ke Za Zhi 2019:21:11-17.

20 WHO. Antimicrobial resistance in the Western Pacific region: a review of surveillance and health system response. Geneva: World Health Organization, 2015.

21 Nguyen PTK, Tran HT, Truong HTT, et al. Paediatric use of antibiotics in children with community acquired pneumonia: a survey from Da Nang, Vietnam. J Paediatr Child Health 2019;55:1329-34 
22 Agweyu A, Lilford RJ, English M, et al. Appropriateness of clinical severity classification of new WHO childhood pneumonia guidance: a multi-hospital, retrospective, cohort study. Lancet Glob Health 2018;6:e74-83.

23 Enarson PM, Gie RP, Mwansambo CC, et al. Reducing deaths from severe pneumonia in children in Malawi by improving delivery of pneumonia case management. PLOS One 2014;9:e102955.

24 Russell FM, Reyburn R, Chan J, et al. Impact of the change in who's severe pneumonia case definition on hospitalized pneumonia epidemiology: case studies from six countries. Bull World Health Organ 2019;97:386-93.

25 Nguyen PTK, Tran HT, Tran TS, et al. Predictors of unlikely bacterial pneumonia and adverse pneumonia outcome in children admitted to a hospital in central Vietnam. Clin Infect Dis 2019;390.

26 Suzuki A, Lupisan S, Furuse Y, et al. Respiratory viruses from hospitalized children with severe pneumonia in the Philippines. BMC Infect Dis 2012;12:267.

27 Althouse BM, Flasche $S$, Minh LN, et al. Seasonality of respiratory viruses causing hospitalizations for acute respiratory infections in children in Nha Trang, Vietnam. Int J Infect Dis 2018;75:18-25.

28 Yoshida L-M, Suzuki M, Thiem VD, et al. Population based cohort study for pediatric infectious diseases research in Vietnam. Trop Med Health 2014;42:S47-58.

29 WHO. Recommendations for management of common childhood conditions: evidence for technical update of pocket book recommendations: newborn conditions, dysentery, pneumonia, oxygen use and delivery, common causes of fever, severe acute malnutrition and supportive care. In: Evidence for technical update of pocket book recommendations. Geneva: World Health Organization, Department of Child and Adolescent Heath and Developement, 2012.
30 Webb BJ, Sorensen J, Jephson A, et al. Broad-spectrum antibiotic use and poor outcomes in community-onset pneumonia: a cohort study. Eur Respir J 2019;54:1900057.

31 Gerber JS, Ross RK, Bryan M, et al. Association of Broad- vs Narrow-Spectrum antibiotics with treatment failure, adverse events, and quality of life in children with acute respiratory tract infections. JAMA 2017;318:2325-36.

32 Song J-H, Jung S-I, Ko KS, et al. High prevalence of antimicrobial resistance among clinical Streptococcus pneumoniae isolates in Asia (an ANSORP study). Antimicrob Agents Chemother 2004;48:2101-7.

33 Van PH, Binh PT, Minh NHL, et al. Results from the survey of antibiotic resistance (SOAR) 2009-11 in Vietnam. J Antimicrob Chemother 2016;71:193-102.

34 Greenberg D, Givon-Lavi N, Sadaka Y, et al. Short-course antibiotic treatment for community-acquired alveolar pneumonia in ambulatory children: a double-blind, randomized, placebo-controlled trial. Pediatr Infect Dis J 2014;33:136-42.

35 Ginsburg AS, Mvalo T, Nkwopara E, et al. Placebo vs amoxicillin for nonsevere fastbreathing pneumonia in Malawian children aged 2 to 59 months: a double-blind, randomized clinical noninferiority trial. JAMA Pediatr 2019;173:21-8.

36 Shrayteh ZM, Rahal MK, Malaeb DN. Practice of switch from intravenous to oral antibiotics. Springerplus 2014;3:717.

37 Addo-Yobo E, Anh DD, El-Sayed HF, et al. Outpatient treatment of children with severe pneumonia with oral amoxicillin in four countries: the MASS study. Trop Med Int Health 2011;16:995-1006.

38 Hadiyono JEP, Suryawati S, Danu SS, et al. Interactional group discussion: results of a controlled trial using a behavioral intervention to reduce the use of injections in public health facilities. Soc Sci Med 1996;42:1177-83. 